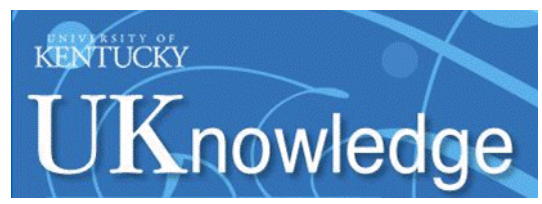

University of Kentucky

UKnowledge

7-28-2014

\title{
Enhanced Charge Photogeneration Promoted by Crystallinity in Small-Molecule Donor-Acceptor Bulk Heterojunctions
}

\author{
Keshab Paudel \\ Oregon State University \\ Brian Johnson \\ Oregon State University \\ Mattson Thieme \\ Oregon State University \\ Michael M. Haley \\ University of Oregon \\ Marcia M. Payne \\ University of Kentucky, marcia.payne@uky.edu
}

See next page for additional authors

Follow this and additional works at: https://uknowledge.uky.edu/chemistry_facpub

Part of the Chemistry Commons

Right click to open a feedback form in a new tab to let us know how this document benefits you.

\section{Repository Citation}

Paudel, Keshab; Johnson, Brian; Thieme, Mattson; Haley, Michael M.; Payne, Marcia M.; Anthony, John E.; and Ostroverkhova, Oksana, "Enhanced Charge Photogeneration Promoted by Crystallinity in SmallMolecule Donor-Acceptor Bulk Heterojunctions" (2014). Chemistry Faculty Publications. 33.

https://uknowledge.uky.edu/chemistry_facpub/33

This Article is brought to you for free and open access by the Chemistry at UKnowledge. It has been accepted for inclusion in Chemistry Faculty Publications by an authorized administrator of UKnowledge. For more information, please contact UKnowledge@lsv.uky.edu. 


\section{Enhanced Charge Photogeneration Promoted by Crystallinity in Small-Molecule Donor-Acceptor Bulk Heterojunctions}

\section{Digital Object Identifier (DOI)}

http://dx.doi.org/10.1063/1.4891758

\section{Notes/Citation Information}

Published in Applied Physics Letters, v. 105, no. 4, article 043301, p. 1-4.

Copyright 2014 American Institute of Physics. This article may be downloaded for personal use only. Any other use requires prior permission of the author and the American Institute of Physics.

The following article appeared in Applied Physics Letters, v. 105, no. 4, article 043301, p. 1-4 and may be found at http://dx.doi.org/10.1063/1.4891758.

Authors

Keshab Paudel, Brian Johnson, Mattson Thieme, Michael M. Haley, Marcia M. Payne, John E. Anthony, and Oksana Ostroverkhova 


\title{
Enhanced charge photogeneration promoted by crystallinity in small-molecule donor-acceptor bulk heterojunctions
}

\author{
Keshab Paudel, ${ }^{1}$ Brian Johnson, ${ }^{1}$ Mattson Thieme, ${ }^{1}$ Michael M. Haley, ${ }^{2}$ Marcia M. Payne, ${ }^{3}$ \\ John E. Anthony, ${ }^{3}$ and Oksana Ostroverkhova ${ }^{1, a)}$ \\ ${ }^{1}$ Department of Physics, Oregon State University, Corvallis, Oregon 97331, USA \\ ${ }^{2}$ Department of Chemistry and Biochemistry, University of Oregon, Eugene, Oregon 97403, USA \\ ${ }^{3}$ Department of Chemistry, University of Kentucky, Lexington, Kentucky 40506, USA
}

(Received 17 June 2014; accepted 20 July 2014; published online 28 July 2014)

\begin{abstract}
We examined sub-nanosecond time-scale charge carrier dynamics in crystalline films of a functionalized anthradithiophene (ADT) donor (D) with three different acceptor (A) molecules. A four-fold enhancement in ultrafast charge carrier separation efficiency was observed in D/A blends with a fullerene acceptor added at 7-10 wt. \% concentrations, whereas a gradual decrease in peak photocurrent amplitude with acceptor concentration was observed with functionalized pentacene and indenofluorene acceptors. The results were directly correlated with the ADT-tri(ethylsilyl)ethynyl-F donor crystallinity. In the bestperforming blends, the presence of crystalline acceptor domains was also established. (C) 2014 AIP Publishing LLC. [http://dx.doi.org/10.1063/1.4891758]
\end{abstract}

Organic semiconductors are of interest due to their potential applications in low-cost, solution-processable, and lightweight (opto)electronic devices. ${ }^{1}$ An important subset of these applications, e.g., solar cells, photodetectors, and photorefractive devices, relies on efficient photoinduced charge carrier generation. A common way of improving charge photogeneration efficiency in organic materials involves creating donoracceptor (D/A) bulk heterojunctions (BHJs), in which charge separation occurs via electron (hole) transfer from the photoexcited donor (acceptor) to acceptor (donor). ${ }^{2}$ Considerable research efforts have been devoted to understanding charge generation in polymer/fullerene $\mathrm{BHJs}^{2}$ and, more recently, in small-molecule BHJs. ${ }^{3-10}$ Currently, the best-performing BHJs involve fullerene acceptors, which have significant drawbacks, and the quest for high-performance non-fullerene acceptors remains open. ${ }^{11-14}$ Given that fullerenes exhibit a range of properties that could be favorable for charge carrier photogeneration (including high electron affinity, good electron transport properties, structure and morphology enabling 3D transport, and availability of several closely spaced excited states that can accept electrons), ${ }^{4,13}$ it is important to establish the most critical properties driving charge photogeneration processes in particular D/A systems. In this Letter, we quantify charge photogeneration processes in organic smallmolecule D/A BHJs with a functionalized anthradithiophene (ADT) derivative as the donor and three different types of acceptors. We combine measurements of transient photocurrent (TPC), photoluminescence (PL), and x-ray diffraction (XRD) with numerical modeling to establish the dominant factor in fast charge carrier photogeneration.

For our study, we chose a fluorinated ADT derivative functionalized with tri(ethylsilyl)ethynyl (TES) side groups, ADT-TES-F (Fig. 1(a)), as the donor. ADT-TES-F exhibits 2D "brick-work" $\pi$-stacking and forms crystalline films characterized by hole mobilities reaching $1.5 \mathrm{~cm}^{2} /(\mathrm{Vs})$ in spin-cast thinfilm transistors, ${ }^{15,16}$ fast charge carrier photogeneration, and

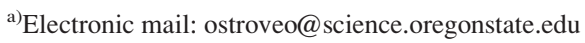

high photoconductive gains. ${ }^{8,17}$ A fluorinated pentacene (Pn) derivative functionalized with tri(isopropylsilyl)ethynyl (TIPS) side groups, Pn-TIPS-F8 (Fig. 1(b)), an indeno[2,1-c]fluorene derivative, indenofluorene (IF)-Mes (Fig. 1(c)), ${ }^{18}$ or $[6,6]-$ Phenyl $C_{61}$ butyric acid methyl ester, PCBM (Fig. 1(d)), were used as acceptors. The choice of the acceptor molecules was guided by the following considerations. Pn-TIPS-F8 and IFMes molecules have similar LUMO energy levels (Fig. 1(e)), which yield D/A LUMO energy offsets of $\sim 0.55 \mathrm{eV}$. (Such offsets should favor high power conversion efficiencies, ${ }^{19,20}$ although the validity of quantitative assessments of the photoinduced charge transfer (CT) efficiency based on this characteristic has been debated in the literature. ${ }^{21}$ ) However, these two molecules exhibit considerably different solid-state packing motifs and excited state dynamics, properties known to affect $\mathrm{CT}$ and charge generation, thus creating different scenarios for photoexcitation relaxation pathways in D/A blends with these acceptors. $^{22}$ In particular, the Pn-TIPS-F8 exhibits 2D "brickwork" $\pi$-stacking (similar to ADT-TES-F) in the solid state, it is fluorescent and, when blended with the ADT-TES-F donor, an emissive CT state (exciplex) with a lifetime of $\sim 4 \mathrm{~ns}$ forms. ${ }^{6,7}$ In contrast, IF-Mes does not yield $\pi$-stacked structures, ${ }^{18}$ and it is non-fluorescent due to fast non-radiative
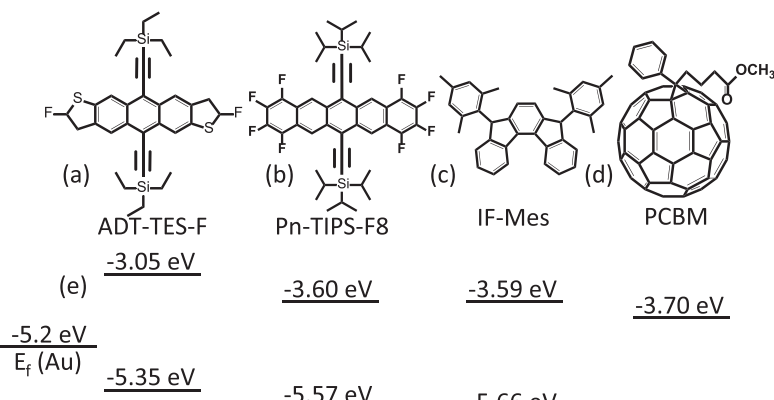

$\underline{-5.35 \mathrm{eV}}$

$\underline{-5.57 \mathrm{eV}} \quad \underline{-5.66 \mathrm{eV}}$

$\underline{-6.10 \mathrm{eV}}$

FIG. 1. (a)-(d) Molecular structures and (e) HOMO and LUMO energies of molecules used in this study. The work function of gold is included for reference. 
relaxation. For comparison, D/A blends with the fullerene acceptor PCBM commonly utilized in organic BHJs were also studied.

In order to prepare samples, $30 \mathrm{mM}$ toluene solutions of donor and acceptor molecules were mixed in appropriate proportions to achieve acceptor concentrations in the D/A films ranging between 0 and 20-40 wt. \%, depending on the acceptor. Films were then spin-cast out of these solutions at $3000 \mathrm{rpm}$, at room temperature, onto glass substrates prepatterned with $\mathrm{Cr} / \mathrm{Au}(5 \mathrm{~nm} / 50 \mathrm{~nm}$ thick) interdigitated electrodes with a finger length and width of $1 \mathrm{~mm}$ and $25 \mu \mathrm{m}$, respectively, and a $25 \mu \mathrm{m}$ gap between adjacent fingers. ${ }^{7}$ Each substrate was also pre-treated with a pentafluorobenzenethiol (PFBT) solution, to facilitate crystallization. ${ }^{16}$ Optical absorption and PL properties of the films can be found in the supplementary material (Figs. S1-S3). ${ }^{23}$

In order to establish the effect of acceptor addition on fast charge carrier photogeneration, we measured timeresolved TPC under a pulsed $532 \mathrm{~nm}$ excitation $(470 \mathrm{ps}$, $55 \mathrm{kHz}$, Q-switched frequency doubled Nd:YAG laser, Altechna STA-01-SH-4-MOPA) at a fluence of $\sim 2.0 \mu \mathrm{J} / \mathrm{cm}^{2}$. Voltage was applied to the samples using a Keithley 237 source-measure unit, and transient photocurrents were measured using a $50 \mathrm{GHz}$ digital sampling oscilloscope (Tektronix CSA8000) with a time resolution of $\sim 0.6 \mathrm{~ns}$ limited by the laser pulse width and jitter. ${ }^{6-9,17}$

TPCs obtained in a pristine ADT-TES-F film and several D/A blends are shown in Figs. 2(a), S4, and S5. Fast photocurrent rise was observed in all samples, regardless of the type of acceptor or its concentration. As shown in Fig. 2(b), D/A films with Pn-TIPS-F8 and IF-Mes acceptors exhibited peak photocurrent amplitudes $\left(I_{p k}\right)$ gradually decreasing as acceptor concentration increased. ${ }^{7}$ In contrast, in ADT-TES-F/PCBM films, $I_{p k}$ increased with PCBM concentration up to $\sim 7-10$ wt. $\%$, reaching values a factor of $\sim 4$ higher than those in pristine ADT-TES-F films, and then decreased at higher concentrations (Fig. 2(b)). Additionally, the initial decay of

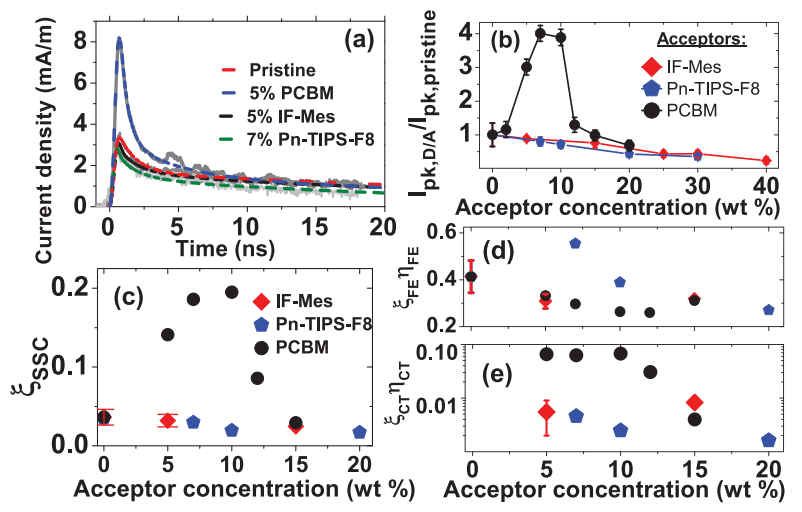

FIG. 2. (a) Transient photocurrents measured in pristine ADT-TES-F donor and various D/A films at $50 \mathrm{kV} / \mathrm{cm}$. Dashed and dotted colored lines represent numerically simulated photocurrents. (b) Peak photocurrent at $50 \mathrm{kV} /$ $\mathrm{cm}$, normalized by that in pristine ADT-TES-F films, as a function of acceptor concentration for the D/A blends with three different acceptors. (c)-(e) Efficiency of (c) ultrafast charge separation $\xi_{S S C}$ and of charge photogeneration via FE (d) and CT exciton (e) dissociation extracted from numerical modeling of the measured photocurrents in various samples. The legends for (d) and (e) are omitted for clarity, but are identical to (c). Error bars are included for several concentrations to illustrate sample-to-sample variation in the fit parameters. the photocurrent in ADT-TES-F/PCBM blends was considerably faster than that in pristine donor films and in D/A films with Pn-TIPS-F8 or IF-Mes acceptors (Fig. 2(a)), due to bimolecular recombination (Fig. S6). Similar trends in both the photocurrent amplitude and decay dynamics were previously observed in ADT-TES-F/ $C_{60}$ films. ${ }^{8,9}$

In order to gain physical insight into charge carrier dynamics in our D/A blends, we performed numerical simulations of the TPC following a previously reported approach, ${ }^{10}$ which has been modified to incorporate a correction ${ }^{24}$ to the Onsager-Braun model. ${ }^{25}$ This enabled us to quantify the distribution of photoexcitation among several relaxation pathways, depending on the acceptor. In our model, charge photogeneration may proceed via (i) ultrafast creation of spatially separated charge carriers (SSCs) (via, for example, band-to-band transitions, hot exciton dissociation, and/or any other process occurring on sub-ps time scales), (ii) Frenkel exciton (FE) dissociation, and (iii) $\mathrm{CT}$ exciton dissociation. The presence of CT excitons was directly confirmed in ADT-TES-F/Pn-TIPS-F8 D/A blends via PL emission from the ADT-TES-F/Pn-TIPS-F8 exciplex centered at $\sim 715 \mathrm{~nm}$ (Fig. S2(a)), as detailed in previous studies. ${ }^{6,7}$ PL emission in ADT-TES-F/IF-Mes and ADT-TES-F/PCBM films was solely due to residual emission from the ADT-TES-F donor (Figs. S2(b) and S2(c)). However, this does not necessarily indicate the absence of CT state formation in these D/A films; for example, dark CT state formation between ADTTES-F and PCBM has been previously inferred from TPC. ${ }^{6,10}$ Thus, the possibility of CT excitons contributing to charge photogeneration was included in our simulations for all D/A blends. The efficiency of the ultrafast channel is characterized by a parameter $\xi_{S S C}$ (which represents the fraction of absorbed photon density that results in SSCs on ultrafast time scales). The parameters of interest for the other two charge photogeneration channels are $\xi_{F E} \eta_{F E}$ and $\xi_{C T} \eta_{C T}$ (where $\xi_{F E, C T}$ is the fraction of absorbed photon density that results in $\mathrm{FE}$ or $\mathrm{CT}$ formation and $\eta_{F E, C T}$ is the $\mathrm{FE}$ or $\mathrm{CT}$ exciton dissociation efficiency over the entire exciton lifetime). We solved numerically a system of coupled differential equations to simulate the photocurrent resulting from the drift of photogenerated charge carriers under applied electric field in the presence of electron and hole trapping, hole detrapping, and Langevin and trap-assisted recombination pathways. ${ }^{10}$ Parameters describing charge photogeneration, transport, and trapping were varied to achieve best possible fits to the experimental data. Examples of fits are included in Figs. 2(a) and S4; contributions of SSC, FE, and CT pathways to TPCs are shown in Fig. S5; fit parameters pertinent to charge photogeneration ( $\xi_{S S C}$ and $\left.\xi_{F E, C T} \eta_{F E, C T}\right)$ are summarized in Figs. 2(c)-2(e); additional fit parameters can be found in Table $\mathrm{S} 1 .^{23}$

These simulations revealed that $\xi_{S S C}$ is the main parameter responsible for the trends in $I_{p k}$ observed in Fig. 2(b). ${ }^{23}$ In ADT-TES-F/PCBM films, $\xi_{S S C}$ increased from $\xi_{S S C}=0.036$ in pristine donor films to $\xi_{S S C}=0.195$ in ADTTES-F/PCBM blends at $10 \mathrm{wt}$ \% PCBM, followed by a decrease at higher concentrations (Fig. 2(c)). In contrast, in composites with Pn-TIPS-F8 and IF-Mes acceptors, $\xi_{S S C}$ gradually decreased with the acceptor concentration, a trend similar to experimental observations in Fig. 2(b). The total 


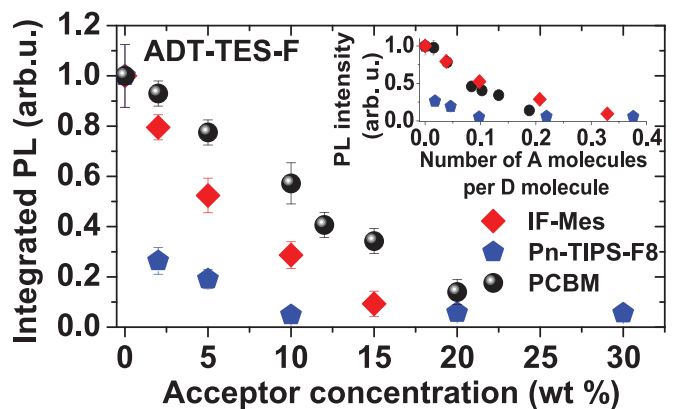

FIG. 3. Integrated PL spectra of the ADT-TES-F donor in D/A blends, normalized by that of pristine ADT-TES-F films, as a function of acceptor concentration. Inset shows the same data re-plotted as a function of the number of acceptor molecules (A) per donor (D) molecule.

contribution of FE dissociation to the photocurrent $\left(\xi_{F E} \eta_{F E}\right.$ in Fig. 2(d)) did not significantly depend on the acceptor, which indicates that this charge generation process occurs inside the ADT-TES-F donor domains and is only slightly affected by the presence of the D/A interfaces. In all D/A blends, the CT exciton dissociation $\left(\xi_{C T} \eta_{C T}\right.$ in Fig. 2(d)) contributed a relatively small percentage of all photogenerated charge carriers (Figs. 2(d) and S5).

Next, we sought a physical explanation for the observed effects of acceptor addition on the ultrafast charge carrier separation efficiency $\xi_{S S C}$ in Fig. 2(c). In order to account for acceptor-dependent differences in, e.g., the extent of the $\mathrm{D} / \mathrm{A}$ interface, D/A packing at the D/A interface, and the density and energetics of the acceptor states, ${ }^{13}$ we quantified donor PL quenching due to CT from the excited donor to acceptor molecules. The integrated donor PL spectra are shown as a function of acceptor wt. \% concentration (Fig. 3, main) and as a function of the number of acceptor molecules per donor molecule (Fig. 3, inset). The conclusions from these plots are consistent: CT was most efficient in the case of the Pn-TIPS-F8 acceptor, the difference especially pronounced at acceptor concentrations below $10 \mathrm{wt}$. \%. This indicates efficient mixing of the Pn-TIPS-F8 and ADT-TESF molecules, facilitated by the similarity of their solid-state packing motifs. Fluorescence imaging of ADT-TES-F/PnTIPS-F8 films supports this observation (Figs. S3(a)-S3(b)). The donor PL quenching was considerably weaker, but followed the same trend, in D/A blends with IF-Mes and PCBM acceptors (inset of Fig. 3), suggesting segregation of $\mathrm{D}$ and A clusters up to at least $15 \mathrm{wt}$. \% acceptor concentration (Figs. S2(c)-S2(d)). Comparison between results in Figs. 2 and 3 suggests that (i) the presence of well-mixed D/ A regions, which leads to exciplex formation in ADT-TESF/Pn-TIPS-F8 blends, does not improve the ultrafast charge carrier separation efficiency $\xi_{S S C}$ and (ii) there is no correlation between the efficiency of CT measured via donor PL quenching (Fig. 3) and of fast charge carrier photogeneration (Fig. 2(b)).

The mechanisms of ultrafast charge carrier separation in organic semiconductors and their D/A blends have been

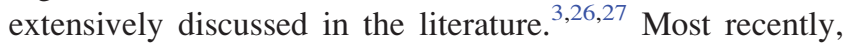
the importance of local crystallinity of donor and acceptor domains, which promote charge delocalization, has been emphasized. ${ }^{2,4,28}$ For BHJs involving polymer donors, crystallinity of acceptor domains, which promote electron

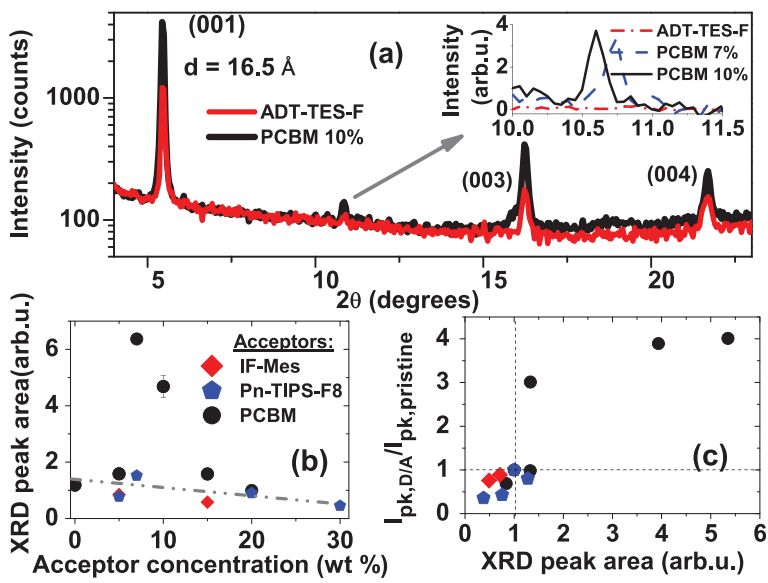

FIG. 4. (a) XRD results for out-of-plane structures in pristine ADT-TES-F and ADT-TES-F/PCBM 10 wt. \% films. Inset shows a magnified view of the peak due to PCBM crystallization in D/A films with $7 \mathrm{wt}$. \% and $10 \mathrm{wt}$. \% of PCBM. Data from a pristine ADT-TES-F film in the same $2 \theta$ region are also included. (b) The (001) donor peak area obtained in different samples. The line provides a guide for the eye. (c) Peak photocurrent normalized by that in pristine ADT-TES-F films as a function of the (001) donor peak area normalized by that in pristine ADT-TES-F films for the D/A blends studied. The legend for (c) is identical to (b).

delocalization specifically, was found to be the dominant factor. In particular, the crystallinity of fullerene acceptor regions occurring at high acceptor loading (typically above 25 wt. \%) has been identified as one of the major reasons for the high performance of fullerenes as acceptors. ${ }^{2}$ In our case, ultrafast charge separation with an efficiency $\xi_{S S C}$ of up to $10 \%$ was observed even in pristine donor films, depending on the film preparation and experimental conditions, ${ }^{10,29}$ and thus far only the addition of a fullerene acceptor has led to improvement in $\xi_{S S C}$, whereas other acceptors inhibited this channel of charge photogeneration. ${ }^{6-9}$ Clues for the cause of this behavior are provided by the XRD data shown in Fig. 4. In all films, peaks at $5.4^{\circ}(001), 16.3^{\circ}(003)$, and $21.7^{\circ}(004)$ were observed, confirming the crystalline nature of our films with a vertical d-spacing of $16.6 \AA$, characteristic of the ADT-TES-F donor (Fig. 4(a)). ${ }^{15,30}$ Addition of Pn-TIPS-F8 and IF-Mes acceptors led to an overall reduction in the crystallinity of ADT-TES-F donor, here quantified by using the area under the (001) peak (Fig. 4(b)), and no formation of crystalline acceptor domains could be detected. Interestingly, addition of PCBM in concentrations of up to 10 wt. \% led to a dramatic enhancement of the donor crystallinity (Fig. 4(b)), ${ }^{31}$ correlated with the enhancement in $I_{p k}$ (Figs. 2(b) and 4(c)). Additionally, in samples with 7 and 10 wt. \% PCBM, a small peak was observed at $10.7^{\circ}$ and $10.8^{\circ}$, respectively (Fig. 4(a), inset), due to crystallization of the PCBM. ${ }^{4}$ As the PCBM concentration further increased, the ADT-TES-F donor crystallinity, as well as $I_{p k}$ (Fig. 4(c)), decreased, and the peak due to PCBM crystallite formation could not be detected. These results may suggest that hole delocalization due to crystallinity of the ADT-TES-F donor is the dominant factor in ultrafast charge carrier separation in pristine ADT-TES-F films and ADT-TES-F-based D/A blends, and that the efficiency of this process can be further improved by electron delocalization in a crystalline acceptor.

In summary, we examined charge carrier photogeneration in small-molecule organic BHJs with a high-mobility 
crystalline donor and three different acceptor molecules. Up to a factor of 4 enhancement in ultrafast charge carrier separation efficiency was observed in $\mathrm{BHJ}$ s with $\mathrm{PCBM}$ added in concentrations of up to $10 \mathrm{wt}$. $\%$. This was attributed to increased donor crystallinity, further assisted by crystallization of the PCBM acceptor, which may enhance hole and electron delocalization, respectively. In contrast, the addition of Pn-TIPS-F8 and IF-Mes led to a reduction in charge carrier photogeneration efficiencies, correlated with decreased donor crystallinity, which may reduce hole delocalization without improving electron delocalization.

We thank A. Fox and Professor B. Gibbons for help with XRD measurements. This work was supported by the NSF Grants DMR-1207309 (O.O.) and CHE-1301485 (M.H.), and the OSU URISC Program (M.T.).

${ }^{1}$ Handbook of organic materials for optical and (opto)electronic devices, 1st ed., edited by O. Ostroverkhova (Woodhead Publishing Limited, Cambridge, UK, 2013).

${ }^{2}$ S. D. Dimitrov and J. R. Durrant, Chem. Mater. 26, 616 (2014).

${ }^{3}$ S. Gélinas, A. Rao, A. Kumar, S. L. Smith, A. W. Chin, J. Clark, T. S. van der Poll, G. C. Bazan, and R. H. Friend, Science 343, 512 (2014).

${ }^{4}$ B. Bernardo, D. Cheyns, B. Verreet, R. Schaller, B. Rand, and N. Giebink, Nat. Comm. 5, 3245 (2014).

${ }^{5}$ L. G. Kaake, Y. Sun, G. C. Bazan, and A. J. Heeger, Appl. Phys. Lett. 102, 133302 (2013).

${ }^{6}$ M. J. Kendrick, A. Neunzert, M. M. Payne, B. Purushothaman, B. D. Rose, J. E. Anthony, M. M. Haley, and O. Ostroverkhova, J. Phys. Chem. C 116, 18108 (2012).

${ }^{7}$ K. Paudel, B. Johnson, A. Neunzert, M. Thieme, B. Purushothaman, M. M. Payne, J. E. Anthony, and O. Ostroverkhova, J. Phys. Chem. C 117, $24752(2013)$

${ }^{8}$ J. Day, A. D. Platt, O. Ostroverkhova, S. Subramanian, and J. E. Anthony, Appl. Phys. Lett. 94, 013306 (2009).

${ }^{9}$ A. D. Platt, M. J. Kendrick, M. Loth, J. E. Anthony, and O. Ostroverkhova, Phys. Rev. B 84, 235209 (2011).

${ }^{10}$ B. Johnson, M. Kendrick, and O. Ostroverkhova, J. Appl. Phys. 114, 094508 (2013).

${ }^{11}$ B. Walker, C. Kim, and T.-Q. Nguyen, Chem. Mater. 23, 470 (2011).
${ }^{12}$ R. J. Kline, S. D. Hudson, X. Zhang, D. J. Gundlach, A. J. Moad, O. D. Jurchescu, T. N. Jackson, S. Subramanian, J. E. Anthony, M. F. Toney, and L. J. Richter, Chem. Mater. 23, 1194 (2011).

${ }^{13}$ T. Liu and A. Troisi, Adv. Mater. 25, 1038 (2013).

${ }^{14}$ M. Schubert, B. A. Collins, H. Mangold, I. A. Howard, W. Schindler, K. Vandewal, S. Roland, J. Behrends, F. Kraffert, R. Steyrleuthner, Z. Chen, K. Fostiropoulos, R. Bittl, A. Salleo, A. Facchetti, F. Laquai, H. W. Ade, and D. Neher, Adv. Funct. Mater. 24, 4068 (2014).

${ }^{15}$ S. K. Park, D. A. Mourey, S. Subramanian, J. E. Anthony, and T. N. Jackson, Appl. Phys. Lett. 93, 043301 (2008).

${ }^{16}$ D. J. Gundlach, J. E. Royer, S. K. Park, S. Subramanian, O. D. Jurchescu, B. H. Hamadani, A. J. Moad, R. J. Kline, L. C. Teague, O. Kirillov, C. A. Richter, J. G. Kushmerick, L. J. Richter, S. R. Parkin, T. N. Jackson, and J. E. Anthony, Nat. Mater. 7, 216 (2008).

${ }^{17}$ A. D. Platt, J. Day, S. Subramanian, J. E. Anthony, and O. Ostroverkhova, J. Phys. Chem. C 113, 14006 (2009).

${ }^{18}$ A. G. Fix, P. E. Deal, C. L. Vonnegut, B. D. Rose, L. N. Zakharov, and M. M. Haley, Org. Lett. 15, 1362 (2013).

${ }^{19}$ T. M. Clarke and J. R. Durrant, Chem. Rev. 110, 6736 (2010).

${ }^{20}$ J. D. Servaites, M. A. Ratner, and T. J. Marks, Eng. Env. Sci. 4, 4410 (2011).

${ }^{21}$ X.-Y. Zhu, J. Phys. Chem. Lett. 5, 2283 (2014).

${ }^{22}$ S. Subramanian, S. K. Park, S. R. Parkin, V. Podzorov, T. N. Jackson, and J. E. Anthony, J. Am. Chem. Soc. 130, 2706 (2008).

${ }^{23}$ See supplementary material at http://dx.doi.org/10.1063/1.4891758 for optical absorption, PL spectra, and PL images as well as details and results of numerical simulations of transient photocurrents.

${ }^{24}$ M. Wójcik and M. Tachiya, J. Chem. Phys. 130, 104107 (2009).

${ }^{25}$ C. L. Braun, J. Chem. Phys. 80, 4157 (1984).

${ }^{26}$ L. G. Kaake, J. J. Jasieniak, R. C. Bakus, G. C. Welch, D. Moses, G. C. Bazan, and A. J. Heeger, J. Am. Chem. Soc. 134, 19828 (2012).

${ }^{27}$ K. Vandewal, S. Albrecht, E. T. Hoke, K. R. Graham, J. Widmer, J. D. Douglas, M. Schubert, W. R. Mateker, J. T. Bloking, G. F. Burkhard, A. Sellinger, J. M. J. Frechet, A. Amassian, M. K. Riede, M. D. McGehee, D. Neher, and A. Salleo, Nat. Mater. 13, 63 (2014).

${ }^{28}$ A. E. Jailaubekov, A. P. Willard, J. R. Tritsch, W.-L. Chan, N. Sai, R. Gearba, L. G. Kaake, K. J. Williams, K. Leung, P. J. Rossky, and X.-Y. Zhu, Nat. Mater. 12, 66 (2012).

${ }^{29}$ B. Johnson, K. Paudel, M. J. Kendrick, and O. Ostroverkhova, Proc. SPIE 8830, 88301S (2013).

${ }^{30}$ Y. S. Chung, N. Shin, J. Kang, Y. Jo, V. M. Prabhu, S. K. Satija, R. J. Kline, D. M. DeLongchamp, M. F. Toney, M. A. Loth, B. Purushothaman, J. E. Anthony, and D. Y. Yoon, J. Am. Chem. Soc. 133, 412 (2011).

${ }^{31}$ A. Sharenko, M. Kuik, M. F. Toney, and T.-Q. Nguyen, Adv. Funct. Mater. 24, 3543 (2014) 